Voix et Images

volxetimages

\title{
Prochain épisode ou la confession manipulée
}

\section{Agnès Whitfield}

Volume 8, numéro 1, automne 1982

Madeleine Gagnon

URI : https://id.erudit.org/iderudit/200369ar

DOI : https://doi.org/10.7202/200369ar

Aller au sommaire du numéro

\section{Éditeur(s)}

Les Presses de l'Université du Québec

\section{ISSN}

0318-9201 (imprimé)

1705-933X (numérique)

Découvrir la revue

\section{Citer cet article}

Whitfield, A. (1982). Prochain épisode ou la confession manipulée. Voix et Images, 8(1), 111-126. https://doi.org/10.7202/200369ar d'utilisation que vous pouvez consulter en ligne.

https://apropos.erudit.org/fr/usagers/politique-dutilisation/ 


\title{
Prochain épisode ou la confession manipulée
}

\author{
par Agnès Whitfield, Université Queen's
}

Livre touffu et déroutant, Prochain épisode continue à résister aux définitions formelles précises. S'agit-il d'une histoire d'amour, d'une "œuvre révolutionnaire»', d'un "produit paradoxal du refus du livre" ${ }^{2}$, ou bien d'un "anti-roman" 3 ? Encore faut-il ajouter aux nombreuses appellations de la critique, celles, tout aussi variées, que propose le narrateur lui-même. Toujours préoccupé par la forme de son livre, il envisage à tour de rôle d'écrire un «roman d'espionnage ${ }^{4}$, un «procès-verbal ${ }^{5}$, des «mémoires» 6 , voire de laisser les "vrais romans aux vrais romanciers". ${ }^{\text {" }}$

Ce n'est pas seulement par leur prolifération que ces étiquettes obscurcissent plus qu'elles n'éclairent la structure formelle nouvelle de Prochain épisode. Le plus souvent elles résultent de jugements intuitifs ou d'analyses de l'anecdote du roman. C'est-à-dire que l'on décide de la forme du livre à partir des réflexions du narrateur sur son "refus du livre» ou sur les grandes questions de l'amour et de la révolution. Seulement, il n'y a pas forcément de rapport d'homologie entre les intentions formelles du narrateur ou le genre d'anecdote qu'il choisit de raconter et la forme de son roman. Celle-ci se définit plutôt par les configurations des traits formels réalisés et surtout, par le rapport entre ces configurations et les procédés formels et les formes romanesques conventionnels. Comme l'a souligné le formaliste russe, Chklovski, «l'œuvre d'art est perçue en relation avec les autres œuvres artistiques et à l'aide d'associations qu'on fait avec elles. Non seulement le pastiche, mais toute œuvre d'art est créée en parallèle et en opposition à un modèle quelconque. ${ }^{8}$

La pertinence de cette constatation de Chklovski est particulièrement évidente dans le cas des romans innovateurs. Par définition même, l'originalité et la nouveauté n'existent que par rapport au connu, à l'habituel. Autrement dit, c'est justement parce qu'il s'écarte des structures ou des procédés conventionnels, qu'un roman est perçu comme étant innovateur. Aussi, ne peut-on cerner la nouveauté formelle d'un tel roman, sans le mettre en rapport avec les formes conventionnelles auxquelles il renvoie.

La complexité de Prochain épisode rend difficile l'identification même de ces modèles formels de repère. Roman en «je», Prochain épisode ne peut renvoyer aux romans en «il», car le choix d'une voix narrative constitue un 
élément important de la construction formelle d'un roman. ${ }^{9}$ La comparaison avec plusieurs formes du roman à la première personne fait aussi ressortir des différences de taille. Bien que le narrateur prône de temps à autre l'incohérence, écho du flux de conscience, le long récit chronologique, voire chronométrique, des aventures du héros en Suisse, trouverait mal sa place dans un monologue intérieur conventionnel. La durée narrative assez courte (de quelques mois à peine avec mise en relief de quelques jours) exclut toute comparaison avec les mémoires. En revanche, nous constatons des recoupements assez poussés avec deux autres formes du roman en «je», soit la confession et ce que $\mathrm{G}$. Genette appelle le «roman du romancier». ${ }^{10}$

Prochain épisode rappelle ce dernier genre romanesque par la distinction que le narrateur tente de maintenir entre lui-même et son héros, qui rédige aussi un livre, et par l'intérêt des deux locuteurs pour les questions de forme littéraire. Nous montrons ailleurs ${ }^{11}$ que la nouveauté de Prochain épisode vis-à-vis du roman du romancier conventionnel tient surtout à la fusion progressive des deux instances narratives et à la nature de leurs objectifs narratifs. Dans un roman du romancier traditionnel, du genre des FauxMonnayeurs, la mise en abyme de l'acte narratif a pour but d'articuler une certaine réflexion critique limitée ${ }^{12}$ sur l'illusion mimétique et la narration romanesque. Pourtant, les deux narrateurs de Prochain épisode visent plutôt à l'affirmation discursive et révolutionnaire. Par conséquent, leur situation de locution est fonction non pas de préoccupations littéraires mais de l'évolution de leur attitude à l'égard de l'anecdote, et plus particulièrement de leur capacité d'assumer la responsabilité de la narration de cette anecdote.

Or, cette notion de responsabilité narrative a aussi des conséquences formelles importantes dans la confession. Notre analyse des écarts entre Prochain épisode et le roman du romancier nous a donc amenée à nous interroger sur la possibilité de recoupements plus profonds avec cette dernière forme romanesque. Le rapprochement avec la confession est d'ailleurs suggéré aussi par d'autres aspects du roman. Quelle que soit leur interprétation de Prochain épisode, les critiques s'accordent en général pour souligner l'identité implicite du narrateur détenu dans un asile psychiatrique à Montréal et du héros révolutionnaire en mission homicide en Suisse ${ }^{13}$. Sous prétexte de présenter l'histoire d'un autre, le narrateur décrit en fait ses propres aventures passées. Aventures que I'on qualifierait mieux de mésaventures, vu la difficulté que le narrateur éprouve à les évoquer et l'importance des thématiques de l'échec, du suicide et de l'hésitation. ${ }^{14}$ On pourrait ainsi dire que Prochain épisode possède, quoiqu'à un niveau implicite, les trois caractéristiques principales de la confession. Un sujet fait lui-même, à la première personne, le récit de ses actions passées, sur lesquelles il porte un jugement défavorable.

Tant de recoupements laissent croire que, dans Prochain épisode, le rôle de modèle de repère revient surtout à la confession. Cette hypothèse, qui peut étonner à première vue, nous semble non seulement valable, mais apte à éclairer bon nombre des éléments formels nouveaux du roman. Nous allons 
montrer que ceux-ci reflètent, en grande partie, une manipulation des procédés traditionnels de la confession.

Pour voir comment Prochain épisode se construit «en parallèle et en opposition» à la confession conventionnelle, il importe de saisir les écarts et les ressemblances a la fois au niveau de ce que J. Starobinski appelle "la discursivité même de la confession, c'est-à-dire la narration d'une suite d'événements dans un enchaînement successif "15 et sur le plan de l'intentionnalité ${ }^{16}$ du sujet qui entreprend de faire cette narration. Dans la confession conventionnelle, il s'agit d'une intentionnalité d'ordre surtout didactique. Comme le remarque N. Frye, la confession résulte presque toujours de l'intérêt théorique ou intellectuel du locuteur pour des questions religieuses, politiques ou esthétiques. "C'est parce qu'il arrive à se faire une vue d'ensemble sur ces questions, que le locuteur croit que sa vie mérite d'être racontée \$17. Le destinataire de la confession est ainsi appelé à participer au jugement que le locuteur porte lui-même sur sa vie ou sur une partie de celle-ci. D'où le caractère à la fois introspectif et rétrospectif du récit des événements. La suite des faits pertinents doit être exposée bien clairement afin que le destinataire puisse en tirer les mêmes conclusions que le locuteur.

De façon schématique, la confession conventionnelle consiste donc en un récit de faits, présenté le plus souvent dans l'ordre chronologique et entrecoupé des commentaires du locuteur, soucieux de transmettre un certain message didactique au destinataire. Pour voir comment ces deux éléments discursif et didactique sont manipulés dans Prochain épisode, nous allons essayer de faire la présentation du récit des faits, c'est-à-dire le déroulement de la mission homicide du révolutionnaire, et ensuite, la façon dont ce récit est pris en charge par les instances du discours, notamment à quelles fins didactiques ou autres.

Tout récit d'événements comporte forcément une double temporalité. Autrement dit, il y a le temps de l'aventure ou de l'anecdote et le pseudo-temps du texte où la succession temporelle des événements anecdotiques est rendue par la successivité spatiale ${ }^{18}$. Deux concepts élaborés par $\mathrm{G}$. Genette nous aideront à préciser le rapport entre ces deux temporalités anecdotique et textuelle dans le récit de la mission homicide du héros de Prochain épisode. II s'agit de repérer ce que Genette appelle les variations dans les rapports d'ordre et de vitesse ${ }^{19}$. Dans les deux cas, l'analyse exige que l'on isole d'abord les grandes articulations de l'anecdote car c'est par rapport à celles-ci que se dégagent les variations d'ordre et de vitesse.

La mission du héros révolutionnaire, que nous désignerons par l'initiale "H», se déroule en sept séquences. Dans une séquence initiale (S1), la maîtresse de $\mathrm{H}, \mathrm{K}$, demande à son amant de tuer un contre-révolutionnaire nommé à tour de rôle von Ryndt, de Heutz et Saugy, en lui laissant entendre qu'il sera récompensé par son amour, s'il s'acquitte de sa mission ${ }^{20}$. H relève le défi et part à la recherche de sa victime. La poursuite de de Heutz fait l'objet de la deuxième séquence $(\mathrm{S} 2)^{21}$. $\mathrm{H}$ finit par dénicher le banquier à Genève mais, au lieu de le tuer, il se fait prendre lui-même. La troisième séquence (S3) 
rend compte de l'évasion de $\mathrm{H}^{22}$ qui parvient à s'accaparer de sa victime, sans toutefois pouvoir l'exécuter. Dans la quatrième séquence (S4), H se prépare à tuer de Heutz au bois de Coppet. Mais le banquier lui raconte une histoire larmoyante; $\mathrm{H}$ compatit avec sa victime et tergiverse de nouveau. ${ }^{23} \mathrm{La}$ cinquième séquence (S5) se déroule au même endroit. De nouveau sur le point de tirer sur de Heutz, $\mathrm{H}$ se fait surprendre par la complice de son adversaire et doit $\mathrm{s}^{\prime}$ enfuir ${ }^{24}$. Dans la sixième séquence (S6), $\mathrm{H}$ décide d'attendre de Heutz dans le château du banquier. L'attente se prolongeant, il en vient presque à mettre en doute sa mission ${ }^{25}$. Mais lorsque de Heutz arrive, il tire sur lui, sans le tuer ${ }^{26}$. Malgré son échec, $H$ se présente au rendez-vous convenu avec $K$, mais trop tard pour y retrouver sa maîtresse. Elle lui laisse cependant un message qui déclenche une septième et dernière séquence (S7). $H$ doit retourner à Montréal pour voir à leurs «intérêts là-bas» 27. Le héros rentre effectivement au Québec où il se fait arrêter et envoyer dans une clinique psychiatrique ${ }^{28}$.

Malgré l'apparence souvent chaotique et peu cohérente de l'ensemble du texte, le récit de la mission de $\mathrm{H}$ s'organise de façon très logique. La première et la dernière séquences servent d'encadrement au récit en précisant pourquoi $H$ entreprend sa mission et quelles en sont les séquelles. Les séquences intermédiaires (S2-S6) portent sur les divers moments-clés de la mission, suivant une gradation bien définie. Il y a d'abord la poursuite (S2) et l'enlèvement (S3) de la victime et ensuite, trois tentatives homicides, chacune plus poussée que la précédente. Lors de la première tentative (S4), $H$ refuse à faire feu sur son otage. La séquence suivante (S5) le voit sur le point de tirer sur de Heutz lorsqu'arrive une complice de ce dernier. $H$ finit par tirer pour de bon sur le banquier (S6).

Pour ce qui est des rapports d'ordre, on pourrait dire que les sept séquences sont présentées en général dans l'ordre chronologique. C'est-ä-dire que l'ordre de disposition des séquences dans le texte respecte l'ordre temporel «réel» des événements ${ }^{29}$. Il y a pourtant deux exceptions à cette synchronie. La première concerne la deuxième tentative manquée de $\mathrm{H}$ au bois de Coppet (S5). Le chapitre IX se termine sur l'image de H, prêt à appuyer sur la gâchette de son Mauser, lorsqu'il est «frappé» d'une «indécision sacrée», plongé «dans une transe profonde ${ }^{30}$. Selon notre découpage de l'anecdote, il s'agit de la fin de S4. Au lieu d'amorcer S5, les chapitres $X$ et $X \mid$ reprennent plutôt la narration des séquences antérieures (S2, S3 et \$4). Lorsque le narrateur revient enfin à $\mathrm{S} 4$ (fin du chapitre $\mathrm{Xl}$ ), l'échec que $\mathrm{H}$ essuie au bois de Coppet est imputé à l'intervention de la complice de de Heutz et non à la défaillance du héros. Du reste, la question de savoir quand et comment $H$ sort de sa transe est laissée en suspens.

L'étroite correspondance entre la présentation des faits dans le récit et leur ordre réel, est bousculée une deuxième fois aux chapitres $X V$ et XVI. Cette fois-ci, c'est la fin de S6 qui est déplacée. Au lieu d'être présentée au chapitre $X V$ après la narration du retour du banquier à son château, la confrontation subséquente entre $H$ et sa victime n'est racontée qu'au 
chapitre XVII alors que les événements de la séquence suivante (S7) nous sont déjà connus.

On remarquera que les deux écarts vis-à-vis de l'ordre chronologique se produisent à un moment où il faut décrire une confrontation entre $\mathrm{H}$ et de Heutz et l'échec subséquent du révolutionnaire. Une coïncidence semblable caractérise les variations dans la vitesse du récit. Genette définit celle-ci comme "le rapport entre une durée, celle de l'histoire, mesurée en secondes, minutes, heures, jours, mois et années et une longueur : celle du texte, mesurée en lignes et en pages. „31 Dans la plupart des récits, le rapport entre la durée de l'histoire et la longueur du récit est loin d'être constant. Certains événements sont présentés de façon très élaborés, ce qui entraîne un «ralentissement» du récit ${ }^{32}$ tandis que d'autres sont racontés de façon sommaire. On parle alors d'accélération du récit. Dans Prochain épisode, les variations de vitesse reflètent systématiquement l'articulation anecdotique. Chaque fois qu'une tentative homicide est imminente, il y a ralentissement du récit, tandis que la narration de la confrontation elle-même et de l'échec subséquent de $\mathrm{H}$ déclenche toujours une accélération du récit.

Entrons dans le détail. Exception faite de la séquence initiale très élaborée, les trois premières séquences (S1, S2 et $\mathrm{S3}$ ) où il n'est question que de la poursuite du banquier et de l'évasion du révolutionnaire, sont racontées suivant une vitesse constante, soit de 0,7 heure/page environ. ${ }^{33}$ Pourtant, à partir du premier attentat de $H$ (S4), les variations de vitesse deviennent importantes. La randonnée en voiture de Genève à Coppet, d'une durée approximative de trois heures, est présentée en quatre pages tandis que l'arrêt d'une demi-heure au bois de Coppet où $\mathrm{H}$ projette de tuer de Heutz, fait l'objet de onze pages. La vitesse du récit passe donc de 0,7 à 0,05 heure/page, à mesure que s'approche le moment de narrer la confrontation entre $\mathrm{H}$ et de Heutz. Par contre, lorsque l'échec subséquent du révolutionnaire est présenté, la tentative manquée est décrite en deux pages et demie seulement, ce qui équivaut à une accélération prononcée du récit, de 0,05 à 0,2 heure/page.

L'attente de $\mathrm{H}$ au château de de Heutz qui précède son dernier attentat contre le banquier ( $\mathrm{S} 6$ ) donne lieu aussi à un ralentissement du récit. Quatre heures sont présentées alors en trente-deux pages pour une vitesse moyenne de 0.01 heure/page. La narration de l'attentat lui-même ne s'étend que sur deux pages, pour une vitesse de 0,1 heure/page. II faut noter par ailleurs que le ralentissement le plus marqué se produit avant la dernière tentative homicide, en l'occurrence la plus importante (S6) tandis que la vitesse maximale est atteinte lors de la narration de l'échec ultime du révolutionnaire, soit son retour au pays (S7). Quelques jours sont résumés alors en cinq pages pour une vitesse d'environ 14,5 heures/page.

Les variations dans les rapports d'ordre et de vitesse ont donc toujours pour effet de remettre à plus tard la narration des échecs de $\mathrm{H}$, ou bien, de permettre un résumé sommaire des faits qui minimise la responsabilité du héros (S4). Elles témoignent de la difficulté qu'éprouve le narrateur à évoquer 
et à assumer les moments les plus pénibles de la mission homicide. Mais la narration de cette mission n'est pas pour autant abandonnée. Au niveau de la présentation du récit des faits, la discursivité de la confession conventionnelle est donc à la fois respectée (l'aveu se poursuit conformément, de façon globale, à l'ordre chronologique et logique) et rejetée (le narrateur tergiverse, résiste à l'aveu).

Cette même tension caractérise également la manière dont le récit des faits est pris en charge par le discours. Traditionnellement, la structure discursive de la confession reflète essentiellement deux facteurs, à savoir le caractère à la fois introspectif et rétrospectif du récit et l'intentionnalité didactique du sujet producteur. Chaque facteur détermine un aspect particulier de la structure discursive conventionnelle par rapport auquel nous pouvons cerner la nouveauté de Prochain épisode.

Du premier facteur résultent un double codage temporel et un écart d'identité chez le sujet producteur. Comme le remarque J. Starobinski, il y a d'une part, les faits passés, objets de la rétrospection, et d'autre part, les commentaires, au présent, du «je actuel», contemporains au discours ${ }^{34}$. Le moi révolu est le personnage principal de l'histoire; la responsabilité ultime du récit incombe au moi actuel, instance principale du discours. C'est ce dernier qui présente l'aventure du moi révolu à l'allocutaire et qui en tire les conclusions qui s'imposent.

Or, cet écart d'identité entre le moi révolu et le moi actuel se retrouve dans Prochain épisode, mais sous une forme équivoque. Le moi révolu est présenté comme le «personnage» 35 du moi actuel ou narrateur, l'identité des deux instances restant implicite jusqu'à la fin du livre. En même temps, le moi actuel se dévêt d'une partie de ses responsabilités discursives en passant la parole à son personnage. La critique s'en tient en général au découpage global de héros et de narrateur proposé par celui-ci, en accordant la priorité discursive au narrateur. ${ }^{36}$ Mais c'est négliger le fait que le héros raconte souvent sa propre histoire et agit, à ce titre, lui aussi comme narrateur.

A la différence de ce qui se passe dans la confession conventionnelle. ces deux procédés - codage fictif du moi révolu et partage de l'initiative discursive - ont pour effet d'accentuer le décalage entre le moi révolu (le héros $\mathrm{H}$ ) et le moi actuel (le narrateur). Plus précisément, ils permettent au narrateur de réduire sa responsabilité à l'égard tant des actions "fictives" confessées, œuvre de son personnage, que de la présentation même de ces actions, qui est remise à un autre. L'écart d'identité, principe important de structuration de la confession, est donc repris, mais dans une optique nouvelle. Qu'il en résulte une véritable manipulation de ce principe ainsi que du double codage temporel qui le sous-tend, voilà ce qui ressort d'une analyse de la situation de discours du héros et du narrateur.

En tant qu'instances discursives, le héros et le narrateur se distinguent au niveau du «ici/maintenant » que pose obligatoirement, suivant E. Benveniste, chaque locuteur en «je».37 Le premier chapitre du roman comporte une 
présentation parallèle de deux «je», dotés chacun d'une situation temporelle et spatiale différente. D'abord, le narrateur, appelons-le « $R »$, dont la situation spatiale, soulignée par l'adverbe «ici», est celle de la clinique psychiatrique, et dont le présent est le temps de l'internement: «J'écris sur une table à jeu [ ...] Je ne sortirai pas d'ici avant échéance ${ }^{38}$. Ensuite, le révolutionnaire $H$, qui se dote d'une situation spatiale sous-marine en Suisse et pour qui le présent, temps de locution, correspond aussi à l'emprisonnement: "ll me reste encore 60 jours de résidence sous-marine avant de retrouver notre étreinte interrompue [...]. D'ici-là, je suis attablé au fond du lac Léman» ${ }^{39}$. Notons que notre découpage suivant des critères proprement discursifs (situation temporelle et spatiale du locuteur) nous amène à associer l'emprisonnement sous-marin à l'instance que l'on désigne communément par le terme de héros, plutôt qu'au narrateur comme le suggérait le parallélisme affectif.

La distinction est importante car elle permet d'établir que le présent discursif de $\mathrm{H}$ est postérieur à son aventure homicide qui a débuté "l'autre soir à Vévey" ${ }^{40}$. C'est dire, si nous faisons abstraction du discours de $R$, que $\mathrm{H}$ adopte, comme le sujet de la confession, un point de vue rétrospectif sur son aventure, et de ce fait, le double codage temporel conventionnel. Exception faite d'un certain tâtonnement au début du chapitre II, il réserve le présent pour ses commentaires ("Le cryptogramme de I'Hôtel de la Paix ne cesse de me fasciner " ${ }^{41}$ ) et raconte sa mission au passé, d'abord au passé composé («à Lausanne j'ai rencontré... ${ }^{42}$ ) et ensuite au passé simple ("J'étais prêt à frapper impatient même quand je vis...»"3). Sur le plan spatial, le passage de l'introspection à la rétrospection se traduit par un va-et-vient entre le lac Léman, lieu du discours, et les différents cantons suisses, lieux de l'aventure passée. Ce codage temporel est d'autant plus significatif que l'intervalle qui sépare le moment du discours et le temps de l'aventure est très court.

Toutefois, à partir du chapitre XIII, la situation temporelle et spatiale de $\mathrm{H}$ change radicalement. II délaisse alors le lac Léman, lieu du discours, au profit des lieux de l'aventure. Quant au codage temporel, il passe du passé simple au présent pour poursuivre le récit de sa mission homicide. Le taux élevé de phrases exclamatives et le recours fréquent aux verbes d'attitude prêteraient à croire qu'il s'agit là d'une transition au présent historique due à l'émotion grandissante de $\mathrm{H}$. Seulement, nous n'y trouvons pas l'arrière-plan passé (à l'imparfait et au plus-que-parfait) qui accompagne en général le présent historique ${ }^{44}$. En fait, le héros semble abandonner l'optique rétrospective pour se placer au moment même de l'aventure. II tente encore d'évaluer, comme avant, ses démarches, mais c'est surtout pour discuter de leur valeur stratégique relative à l'accomplissement de sa mission, comme si la suite des événements lui était inconnue:

$\mathrm{H}$ de Heutz va bientôt ouvrir la porte à deux vantaux. [...] Aucun bruit ne témoigne encore que $H$ de Heutz est rendu sous le porche. Je n'entends strictement rien, et cela n'est pas sans m'inquiéter. J'aurais mieux fait d'ouvrir le jour du larmier, ce qui m'aurait permis d'entendre ce qui se passe dehors... ${ }^{45}$ 
Chose curieuse, la structure temporelle de la confession conventionnelle, refusée d'abord par $R$, refait surface dans le discours de $H$, où elle est de nouveau rejetée. $H$ adopte initialement un point de repère, le lac Léman, qui lui permet une perspective rétrospective et critique sur son aventure. Mais, par la suite, il abandonne la rétrospection pour s'identifier de plus en plus aux lieux de sa mission. Parallèlement, l'introspection perd toute valeur analytique. Elle est récupérée par les réflexions stratégiques du héros.

La situation spatiale et temporelle de $R$ subit une évolution semblable. Tout en attribuant ses mésaventures passées à son personnage, $R$ effectue néanmoins, au début du livre, quelques petits retours en arrière à son propre compte. Ces esquisses de rétrospection, qui concernent une liaison amoureuse, sont commentées par $\mathbf{R}$ qui souligne leur nature culpabilisante ainsi que son désir de les oublier:

Je parviendrai ainsi jusqu'au coucher, édifiant sans continuité des plans de roman, multipliant les inconnues d'une équation fictive et imaginant, somme toute, n'importe quoi pourvu que cet investissement désordonné me soit rempart contre la tristesse et les vagues criminelles qui viennent me briser avec fracas, en scandant le nom de la femme que j'aime.

Une journée d'hiver, en fin d'après-midi, nous avons roulé... ${ }^{46}$

D'emblée, la fiction se présente donc à $R$, et ce, sur le plan explicite, comme un moyen d'éviter la confession et l'analyse de ses mésaventures passées.

Mais comme dans le discours de $\mathrm{H}$, et de façon plus rapide, la double structure introspective et rétrospective de la confession conventionnelle est évoquée et aussitôt rejetée. $R$ abandonne la rétrospection au profit de ce que Genette appélle la narration simultanée ${ }^{47}$. Le double codage temporel se voit remplacer par le recours exclusif au présent, sans que le potentiel introspectif de ce temps soit davantage exploité. Le contenu anecdotique des retours en arrière est assumé par $\mathrm{H}$ comme en témoignent la corrélation étroite entre la diminution des allusions de $\mathbf{R}$ à son amour et l'augmentation des mentions de $\mathrm{K}$, maîtresse de $\mathrm{H}^{48}$, ainsi que nombreuses observations de $R$ sur le rapport entre son roman et ses souvenirs. ${ }^{49}$ Dans son discours de romancier, $R$ se contente de commenter ses démarches et ses insatisfactions littéraires:

Nul dévergondage scripturaire ne peut plus me masquer le désespoir incisif que je ressens devant le nombre de variables qui peuvent entrer dans la composition d'une cuvre originale. Mais pourquoi suis-je à ce point sensible à ce problème de l'originalité absolue? ${ }^{50}$

Paradoxalement, si la modification de la situation temporelle de $R$ lui permet de se distancier par rapport à sa propre rétrospection, remise à $H$ et fictionnalisée, l'évolution de sa situation spatiale entraîne une identification croissante avec le texte, lieu de cette fiction. Bien que $R$ ne bouge pas de la clinique psychiatrique, son «ici» au moment de locution, le référent de cet 
adverbe se modifie au cours du livre. Au chapitre initial, il désigne six fois la clinique et une fois une phrase d'un livre de Balzac ${ }^{51}$. Jusqu'au chapitre $X$, à cette exception près, l'adverbe renvoie toujours à la clinique ${ }^{52}$. Pourtant, à partir du chapitre $X$, il renvoie au texte même: «Rien n'est libre ici, ni mon coup d'âme, ni la traction adipeuse de l'encre sur l'imaginaire» 53 .

Les rapports que $R$ entretient avec ses souvenirs paraissent ainsi contradictoires. D'une part, il se défait de son passé, en abandonnant toute tentative personnelle de rétrospection et en passant ses aventures à $\mathbf{H}$. D'autre part, par son identification croissante avec le texte, il s'associe étroitement à l'histoire de son personnage, d'autant plus que celle-ci se raconte, comme les réflexions littéraires de $\mathrm{R}$, au présent. Cette contradiction s'accentue dans le discours de l'instance, appelons-la $H / R$, qui représente la fusion de $H$ et de $R$ à la fin du livre.

$H / R$ reprend l'équivalence établie par $R$ entre le texte et son «ici» du locuteur: "je coule ici dans un plasma de mots"54; "J'étouffe ici dans la contre-grille de la névrose, tandis que je m'enduis d'encre»55. De même, le codage temporel adopté par $H / R$ renforce le caractère essentiellement textuel de cette instance narrative. Les quelques instants qui précèdent la dernière confrontation entre $\mathrm{H}$ et de Heutz sont présentés au présent: "Sur le point de bondir, j'attends interminablement le bon moment, le doigt appuyé sur la gâchette. D'un instant à l'autre, je vais sûrement trouver le mot qui manque pour tuer $\mathrm{H}$ de Heutz» ${ }^{56}$. C'est l'allusion au «mot qui manque» qui indique que le rapport entre l'instance discursive et son histoire est d'ordre textuel. Par contre, la confrontation proprement dite entre $\mathrm{H}$ et de Heutz est racontée comme un retour en arrière de $\mathrm{H}(« \mathrm{H}$. de Heutz s'est aperçu de ma présence. Deux balles ont effleuré les moulures de la crédence Henri II, avant même que je me sois rétabli pour contre-attaquer ${ }^{57}$ ), entrecoupé des commentaires stratégiques caractéristiques du discours du héros ("Si je n'avais pas épuisé mes forces à attendre $\mathrm{H}$. de Heutz, je l'aurais tué avec précision» ${ }^{58}$ ). Cependant, pour narrer les séquelles de l'échec de $H$, $H / R$ passe au futur fictif : "Quand les combats seront terminés [...] je trouverai peut-être le temps de mettre un point final à ce livre et de tuer $\mathrm{H}$. de Heutz une fois pour toutes. 59

L'écart d'identité entre $H$ et $R$ est donc résolu à la fin du roman mais par le truchement du texte. C'est la conséquence logique des processus d'identification sous-jacents tant aux rapports de $H$ avec sa rétrospection réactualisée quaux relations ambiguës entretenues par $R$ avec le passé de son personnage. La rétrospection garde șon caractère fictif; le présent de l'introspection est récupéré par des considérations stratégiques découlant de la réactualisation de la mission de $\mathrm{H}$. Notons, toutefois, que l'échec ultime de $H$ n'est pas assumé par $H / R$ qui choisit de le présenter sous forme d'une rétrospection fictive de $H$. Aussi, les conséquences de cet échec pour $H / R$, sont-elles surmontées par un saut dans le futur, également fictif: "Voilà comment j'arriverai à ma conclusion. Oui, je sortirai vainqueur de mon intrigue, tuant $\mathrm{H}$. de Heutz avec placidité» 60 . 
Par ce saut dans le fictif s'effectue une inversion complète de l'écart d'identité de la confession conventionnelle. Le moi actuel, traditionnellement responsable du discours, se fond dans le moi révolu et se fictionnalise. C'est le moi révolu fictif qui assume l'initiative discursive. $R$ arrive ainsi non seulement à nier toute responsabilité à l'égard de ses mésaventures passées, mais aussi à se créer une nouvelle version fictive de son passé, et plus précisément à occulter son échec.

Tant au niveau discursif que sur le plan de la présentation des faits, c'est donc l'échec de $H / R$ qui empêche l'exploitation conventionnelle des procédés de la confession. Ainsi, peut-on s'attendre que le romancier s'écarte aussi de celle-ci par son intentionnalité. Traditionnellement didactique, cette intentionnalité se manifeste par les déclarations d'intention du locuteur et par ses rapports avec son allocution. Dans Prochain épisode, les déclarations de $R$ sont nombreuses et contradictoires. Nous avons vu que sa décision de fuir les conséquences de sa mésaventure en la fictionnalisant, est délibérée. Mais ce n'est pas son seul but. $R$ tient aussi, dit-il, à écrire un roman d'espionnage pour se divertir ${ }^{61}$, à plaire à sa maîtresse en rédigeant une grande lettre d'amour ${ }^{62}$, à éviter la «lucidité homicide» par une écriture automatique ${ }^{63}$ et à contester, par un plaidoyer révolutionnaire, «l'expertise psychiatrique" à laquelle il est soumis ${ }^{64}$. Malgré leur diversité, ces projets se regroupent autour de deux buts principaux, à savoir: 1) la fuite du passé ou de la lucidité, et 2) la contestation politique, la réconciliation amoureuse étant tributaire de l'affirmation révolutionnaire.

C'est le désir de fuir qui prime chez $R$ dans les dix premiers chapitres du livre, sans qu'il arrive à l'accomplir. D'une part, que $\mathbf{R}$ se lance dans un roman d'espionnage ou l'écriture automatique, son passé ressurgit:

Rien n'est libre ici [...] Pas même l'intrigue, ni l'ordre d'allumage de mes souvenirs [...] On dirait que mon passé antérieur a tracé mon cheminement et proféré les paroles que je crois inventer. ${ }^{65}$

D'autre part, la remémoration, ainsi rejetée, se trouve aussi sollicitée par $R$. Les formes romanesques auxquelles il fait allusion ont toutes un coefficient mnémonique: «écriture automatique»66, «mémoires» 67 , « rétrospection» 68 . II en va de même du nouveau roman d'espionnage envisagé par R:

Je suis le cours et ne l'invente jamais. Ceci vaut pour tout ce que j'écris [...] Je risque tout à m'avouer le sujet de mon hésitation. Ce n'est plus l'originalité opératoire de la littérature que je désamorce, c'est l'existence individuelle, qui éclate soudain et me désenchante. ${ }^{69}$

Cette incapacité de $\mathbf{R}$ de renoncer à la remémoration, quand bien même il la récuse, tient au lien étroit qui existe, à ses yeux, entre son passé et ses aspirations révolutionnaires. Temps de l'échec, le passé est aussi l'époque de l'affirmation rebelle, de l'identification aux "patriotes» 70: «Pendant des années, j'ai vécu aplati avec fureur. [...] introduire le lance-flamme en dialectique, et la conduite-suicide en politique, voilà comment j'ai établi mon style» ${ }^{71}$. Refusant d'entrevoir un avenir révolutionnaire réel, écho de 
ce passé rebelle, $R$ s'enlise dans un cercle vicieux. Si l'affirmation révolutionnaire ne passe que par la remémoration, le passé, lui, n'aboutit qu'à l'échec:

En moi, déprimé explosif, toute une nation s'aplatit historiquement et raconte son enfance perdue, par bouffées de mots bégayés et de délires scripturaires et, sous le choc noir de la lucidité, se met soudain à pleurer devant l'immensité du désastre et l'envergure quasi sublime de son échec. ${ }^{72}$

L'importance structurale de ce cercle vicieux est évidente si nous nous rappelons que c'est justement dans les dix premiers chapitres du roman que, à tour de rôle, $R$ appelle et rejette les procédés conventionnels de la confession. De même, la solution adoptée par $R$ pour sortir de l'impasse présage les processus d'identification textuelle qu'il entreprend par la suite:

Ce livre défait me ressemble. [...] il épouse la forme même de mon avenir: en lui et par lui je prospecte mon indécision et mon futur improbable. [...] La seule forme que je poursuis confusément depuis le début de cet écrit, c'est la forme informe qu'a prise mon existence emprisonnée [...] Événement nu, mon livre m'écrit. ${ }^{73}$

La récupération du "livre défait» par la survalorisation de l'écriture, devenue l'avenir même du locuteur, annonce la sublimation fictive subséquente de l'échec de R. Cessant d'être contradictoires, les deux intentionnalités de $R$, la fuite et l'affirmation révolutionnaire, se fondent pour se réaliser dans un «prochain épisode».

But personnel, la fuite de l'échec par l'affirmation révolutionnaire future, ne semble pas correspondre aux objectifs didactiques du je-locuteur de la confession conventionnelle. En occultant son échec, $R$ se dispense de porter un jugement lui-même sur son passé, et par conséquent, de faire partager ce jugement par son allocutaire. II s'ensuit une manipulation du rôle de celui-ci, qui s'effectue, tout comme l'inversion du principe de l'écart d'identité, par le biais de la fiction.

Dans la confession conventionnelle, l'écart d'identité chez le sujet se reproduit dans le double rôle de l'allocutaire. Le jugement du locuteur sur son moi révolu est sanctionné par un allocutaire-juge (voir l'invocation de Dieu dans les Confessions de St-Augustin) garant de la "véracité " du récit et transmis, sous forme de message didactique, à un allocutaire-égal, qui légitimise la "discursivité» de la confession 74. Or, dans Prochain épisode, $\mathbf{R}$ renie le potentiel critique de deux instances, son alter ego et sa maîtresse, qui auraient pu lui servir de conscience. Loin d'assurer la véracité du récit de $R$, ces allocutaires sont appelés seulement à en trancher la forme littéraire. Sauf pour quelques déclarations d'amour au début du livre ${ }^{75}, \mathrm{R}$ s'adresse surtout à sa maîtresse, révolutionnaire par excellence, pour lui faire le récit de ses frustrations de romancier ("Quelques instants avant le fracas, je circulais aimablement dans mon inventaire: j'évoquais les villages que nous avons traversés [...] me voilà terrassé " $\left.{ }^{76}\right)$. A son alter ego, $R$ pose surtout des questions sur la forme romanesque à adopter: «je me pose des questions. Écrire un roman d'espionnage comme ou en lit, ce n'est pas 
loyal» 77 ; «Mais pourquoi suis-je à ce point sensible à ce problème d'originalité absolue? Je ne sais pas» 78 .

Toutefois, dans un premier temps, le potentiel critique de ces deux allocutaires refait surface, comme d'autres éléments conventionnels, dans le discours de $\mathrm{H}$. C'est le héros de $\mathrm{R}$ qui tient, quoique de façon ambiguë, à se faire pardonner son échec par K ( «Tu me détesteras si tu apprends ma faiblesse, la voici quand même» ${ }^{79}$ ) et à s'assurer son amour ( Penses-tu à moi ?» ${ }^{80}$ ). C'est aussi $\mathrm{H}$ qui appelle son alter ego à juger son aptitude à bien remplir sa mission homicide: "Somme toute, je me suis fait disqualifier par $\mathrm{H}$. de Heutz et si j'évite une rétrospection minutieuse de ma mission, c'est pour ne pas tourner le fer dans la plaie ${ }^{81}$.

Mais par la suite même les allocutaires de $\mathrm{H}$ sont récupérés par la fiction. K, maîtresse perdue, sera retrouvée la "prochaine fois" 82 ; l'alter ego juge, non les qualités révolutionnaires de $\mathrm{H}$, mais la vraisemblance de son histoire ( Plus je repense à ces quelques minutes, plus je m'interroge sur la vraisemblance de cette séquence» $\left.{ }^{83}\right)$. Parallèlement, $R$ finit aussi par associer sa maîtresse au «prochain épisode" où elle servira de garante de la foi révolutionnaire du locuteur: “Mon amour, tu m'es sol natal que je prends à pleines mains, sol obscur fuyant que je féconde et où je me bats à mourir, inventeur orgueilleux d'une guérilla infinie» ${ }^{84}$. De son rôle initial de juge littéraire, l'alter ego de $\mathbf{R}$ ne gardera que la possibilité de confirmer les déductions de $\mathbf{R}$ : "Mon écriture témoigne d'une genèse seconde [...]. Donc ma torpeur n'est rien ${ }^{85}$.

Quant à l'allocutaire-lecteur, objet du message didactique dans la confession conventionnelle, lui aussi est appelé par $\mathrm{R}$ à participer à ses préoccupations littéraires. II sert à garantir moins la discursivité du récit de $\mathbf{R}$ que son intérêt littéraire. C'est à ce lecteur virtuel que $R$ se présente, décrit sa situation et annonce ses intentions ${ }^{86}$. Ensuite, il l'invite à réfléchir avec lui sur la forme de son livre: "J'éprouve une grande sécurité, aussi bien l'avouer, à me pelotonner mollement dans le creuset d'un genre littéraire aussi bien défini " ${ }^{87}$; “Bien sûr, j'ai l'air de forcer un peu la note ${ }^{88}$. À partir du chapitre $X$, où $R$ opte pour une œuvre révolutionnaire, le rôle de l'allocutaire-lecteur se trouve davantage manipulé. Gratifiée d'une identité de plus en plus explicitement québécoise ${ }^{89}$, transformé en frère-patriote, l'allocutaire-lecteur doit sanctionner non plus la valeur littéraire du livre de $R$, mais sa portée révolutionnaire: "Il faudra remplacer les luttes parlementaires par la guerre à mort" ${ }^{90}$; "Ce jour-là [de la révolution] les pages s'écriront d'elles-mêmes à la mitraillette: les mots siffleront au-dessus de nos têtes" ${ }^{91}$.

De nouveau, tout procédé qui peut obliger $\mathrm{R}$ à assumer son échec est rejeté. Aucun allocutaire-juge potentiel ne garde son rôle de garant de la véracité du récit de $\mathrm{R}$. Tout au plus, l'alter ego du romancier assure-t-il la vraisemblance de ce récit. Si l'allocutaire anonyme reste témoin, du moins dans un premier temps, de la discursivité de la confession traditionnelie, il finit par être sollicité par l'élan révolutionnaire de R. II devient alors l'objet non plus d'une démarche didactique mais d'un acte de persuasion. 
Nul n'est besoin d'insister davantage sur l'importance, dans Prochain épisode, des procédés de construction de la confession conventionnelle. Trois de ces procédés sont repris sous une forme inversée. L'écart d'identité et la double temporalité qui affirment traditionnellement la précession du moi actuel, sont supprimés au profit du moi révolu, révolutionnaire. Au lieu de précéder la rédaction, en tant que gage de la responsabilité qu'assume le moi actuel pour son passé, la fusion de ces instances s'accomplit à la fin du livre dans un contexte fictif où le méfait passé est récupéré par un futur glorieux. Même inversion au niveau de l'intentionnalité. La volonté d'avouer cède la place au désir de fuir; le respect des valeurs traditionnelles, à la contestation révolutionnaire. En revanche, deux procédés conventionnels sont retenus sous une forme modifiée, à savoir la discursivité et la démarche didactique, transformée en acte de persuasion.

Que ce soit en opposition, par l'inversion, ou en parallèle, par l'exagération, $R$ ne cesse donc de définir son texte par rapport à la confession. Si l'inversion de l'écart d'identité, de la double temporalité et de l'intentionnalité conventionnelles, s'expliquent par le désir de $\mathrm{R}$ d'occulter son échec, la reprise de la discursivité et, de façon exagérée, la démarche didactique, paraît quelque peu paradoxale. En effet, rien n'empêche $R$ d'abandonner le récit de sa mission manquée, tout comme il en refuse la responsabilité discursive. II sortirait alors carrément du contexte de la confession, éliminant ainsi la nécessité de prendre parti face à son échec, tant à ses propres yeux qu'à ceux d'autrui. Cette démarche lui est pourtant interdite. tant qu'il tient non seulement à occulter son échec mais aussi à établir une nouvelle version fictive de son passé. Remise en question, tant qu'elle menait à l'échec, la discursivité de la confession conventionnelle devient la garante même de cette nouvelle perspective fictive. Seule la chronologie passée permet d'envisager et de légitimer un "prochain épisode».

Pour la même raison, $\mathbf{R}$ doit manipuler plutôt que supprimer le rôle conventionnel de l'allocutaire-témoin. Faute de pouvoir prouver la véracité du prochain épisode, il doit à plus forte raison, en assurer la vraisemblance. C'est dans la mesure où son lecteur virtuel se laissera persuader par son élan révolutionnaire que $\mathrm{R}$ peut y croire lui-même. Manipuler la confession, pour se laisser ensuite manipuler par elle, voilà le tour de force du narrateurromancier de Prochain épisode. Tout de force pour lequel l'envoûtement et la confusion de la critique ne constituent pas de minces hommages. Comment voir, en effet, sous l'emprise d'un tel "prochain épisode», que la nouveauté formelle du livre réside dans une simple mais habile manipulation d'une forme romanesque ancienne: la confession?

1. Smart, P., Hubert Aquin, agent double, Montréal, Presses de I'Université de Montréal, 1973, p. 10.

2. Lefebvre, J., "Prochain épisode ou le refus du livre», Voix et Images du Pays, V (1972), p. 163.

3. Smart, P., op. cit., p. 25. 
4. Aquin, H., Prochain épisode, Montréal, Le Cercle du livre de France, 1965, p. 7.

5. Ibid., p. 45.

6. Ibid., p. 25 .

7. Ibid., p. 14.

8. Chklovski, V., in Todorov, T., Théorie de la littérature, Paris, Seuil, 1965, p. 50.

9. Butor, M., Essais sur le roman, Paris, Editions de Minuit, 1960, p. 75.

10. Genette, G., Figures III, Paris, Seuil, 1972, p. 237.

11. Whitfield, A., La problématique de la narration dans le roman québécois écrit a la première personne depuis 1960, Thèse de doctorat, Université Laval, 1981. pp. 404-414.

12. Voir, entre autres, $M$. Raimond qui souligne la portée critique de la mise en abyme de l'acte narratif dans le roman du romancier (Le roman contemporain, le signe des temps, Paris, CEDES, 1976, p. 118) et J. Verrier qui fait remarquer que Gide crée une autre illusion tout en mettant en cause l'illusion mimétique traditionnelle (“Le récit réfléchi», Littérature, 5 (février, 1972), p. 59).

13. Voir, entre autres, Smart, P., op. cit. ; Legris, R., «Structures de l'action romanesque dans Prochain épisode», in R. Quellet, Le Nouveau roman, Editions Garnier, Paris, 1972, pp. 152-157; Lasnier, L., «Spatio-analyse de Prochain épisode, Québec littéraire, 2 (1976), pp. 33-54; Melançon, J., "Le procès métaphorique dans Prochain épisode, Québec littéraire, 2 (1976), pp. 15-24.

14. Voir, entre autres, Smart, P., op. cit.; Bouthillette, J., "Écrivain, faute d'être banquier", in Aquin, H., Point de fuite, Montréal, Cercle du livre de France, 1971. pp. 13-20; Berthiaume, A., "Le thème de I'hésitation dans Prochain épisode». Liberté XV, 1 (janvier-février 1973), pp. 135-148; Lefebvre, J., loc. cit.; de la Fontaine, G., Hubert Aquin et le Québec, Montréal, Parti Pris, 1971.

15. Starobinski, J., “Le Style autobiographique», Poétique, 3 (1970), p. 257.

16. Nous n'entendons pas par ce terme l'intention psychologique du sujet, mais ses buts narratifs, tels qu'ils se révèlent dans le texte, à travers la situation discursive du sujet producteur.

17. Frye, N., Anatomy of Criticism. Princeton, Princeton University Press, 1957, pp. 307-308.

18. Genette, G., Figures III, op. cit., p. 77.

19. Lbid.

20. Aquin, H., Prochain épisode, op. cit., p. 42.

21. Ibid., pp. 45-55.

22. Ibid., pp. 57-74.

23. Ibid., pp. 75-88.

24. Ibid., pp. 105-107.

25. Ibid., pp. 107-146.

26. Ibid., pp. 147-149.

27. Ibid., p. 158.

28. Ibid., p. 164.

29. Genette. G., op. cit., p. 78.

30. Aquin, H., Prochain épisode, op. cit., p. 88.

31. Genette, G., op. cit., p. 123.

32. Ibid., p. 127.

33. La poursuite du banquier qui s'étend sur 7,5 heures (de $18 \mathrm{~h} 30$ à $2 \mathrm{~h}$ du lendemain) est racontée en 8 pages. L'emprisonnement du héros et son évasion s'étendent sur 4 heures $(5 \mathrm{~h} 30$ à $9 \mathrm{~h} 30)$ et sont décrits en 9 pages. La vitesse moyenne pour les deux séquences est donc de 0.7 heure/page.

34. Starobinski, J., lo. cit., p. 261.

35. Aquin, H., Prochain épisode, op. cit., p. 96. Le narrateur parle aussi de son «délégué de pouvoir» (Ibid., p. 47). 
36. Voir, entre autres, Legris, R., loc. cit.; Smart, P., op. cit.; Melançon, J., loc. cit.

37. Benveniste, E., «L'Appareil formel de l'énonciation», Langages, 17 (mai 1970). pp. 13-15.

38. Aquin, H., Prochain épisode, op. cit., p. 7.

39. Ibid., p. 11.

40. Ibid.

41. Ibid., p. 21.

42. Ibid., p. 29.

43. Ibid., p. 53

44. Weinrich, H., Le Temps, Paris, Seuil, 1973, p. 305.

45. Aquin, H., Prochain épisode, op. cit., p. 147.

46. Ibid., p. 10.

47. Genette, G., op. cit., p. 229.

48. Après avoir interpellé sa maîtresse au chapitre initial, $R$ la délaisse jusqu'au chapitre VIII. Par contre, $H$ parle souvent à $\mathrm{K}$ aux chapitres I, II, III, VII et VIII. De même, à partir du chapitre VIII, l'importance de la maîtresse de $R$ va en décroissant, et celle de l'amie de $\mathrm{H}$, en augmentant.

49. Aquin, H., Prochain épisode, op. cit., pp. 47, 71, 90, 91.

50. Ibid., p. 91.

51. Ibid., pp. 7, 9, 11, 13, 14, 15, 16.

52. lbid., pp. 35, 47 (2 mentions), 71, 73.

53. Ibid., p. 89. Voir aussi lbid., p. 93.

54. Ibid., p. 164.

55. Ibid.

56. Ibid., p. 165.

57. lbid., pp. 165-6.

58. lbid., p. 170.

59. Ibid., p. 173.

60. Ibid.

61. Ibid., p. 9

62. Ibid., p. 70 .

63. Ibid., p. 13.

64. Ibid., pp. 24-25.

65. Ibid., pp. 89-90.

66. Ibid., p. 25.

67. Ibid.

68. Ibid., p. 60 .

69. Ibid., p. 91.

70. Ibid., p. 70.

71. Ibid., p. 24.

72. Ibid., p. 25.

73. Ibid., pp. 92-94.

74. Starobinski, J., loc. cit., p. 261.

75. Lid., pp. 7-8.

76. Ibid., p. 71.

77. Ibid., p. 9.

78. Ibid., p. 91.

79. Ibid., p. 40 .

80. Ibid., p. 73 .

81. Ibid., p. 60 
126 - VOIX ET IMAGES, VOL. VIII, NO 1

82. Jbid., p. 153.

83. Ibid, p. 98.

84. Jid., p. 143.

85. Thid., p. 119.

86. Ibid., pp. 8-9.

87. Ibid., p. 8.

88. Ibid.

89. Ibid., pp. 78, 94, 120.

90. Ibid., p. 172.

91. Ibid. 